\title{
Réalisabilité d'un modèle non-linéaire de la corrélation pression-température
}

\author{
Samira Gaaliche ${ }^{\mathrm{a}}$, Mounir Bouzaiane et Taieb Lili \\ Département de Physique, Faculté des Sciences de Tunis, Campus Universitaire, 2092 El Manar, Tunisie
}

Reçu le 28 juin 2004, accepté le 29 juillet 2005

\begin{abstract}
Résumé - Dans ce travail, nous proposons un modèle non-linéaire de retour à l'isotropie de la corrélation pression-température suivant un développement à ordre cinq du tenseur anisotrope de Reynolds $\vec{b}$. Ce modèle est un essai d'extension du modèle récent de Chung et Kim pour la corrélation pression-déformation. L'étude de stabilité des états d'équilibre d'une turbulence homogène évoluant en l'absence de gradients moyens, conduit à une condition simple sur l'un des coefficients du modèle proposé. De plus, une étude de réalisabilité est effectuée. Nous montrons qu'une condition suffisante de réalisabilité du modèle proposé est obtenue en exprimant ses coefficients en fonction de ceux du modèle de la corrélation pression-déformation. Enfin, une simulation numérique sur la base de résultats de simulation numérique directe de Iida et Kasagi a montré que le modèle proposé assure une meilleure prédiction de la turbulence thermique par rapport au modèle classique de Rotta.
\end{abstract}

Mots clés : Retour à l'isotropie / corrélation pression-température / stabilité des points fixes / réalisabilité

\begin{abstract}
In this work, we propose a non linear model for the return to isotropy term of the pressuretemperature correlation as a development of a fifth power of the Reynolds stress anisotropy $\vec{b}$. This model is an essay of extension of the recent model of Chung \& Kim that is retained for the slow pressure-strain correlation. The study of the stability of the equilibrium states of a homogeneous turbulence, in the absence of mean gradients, leads to a simple condition on one of the coefficients of the proposed model. Moreover, a study of realisability has been developed. We demonstrate that a sufficient condition of realisability of the proposed model is obtained when its coefficients are expressed according to those of the pressure-strain correlation model. Finally, a numerical simulation on the basis of the direct simulation results of Iida \& Kasagi, has shown that the proposed model ensures a better prediction of the thermal turbulence with respect to Rotta's classic model.
\end{abstract}

Key words: Return to isotropy / pressure-temperature correlation / fixed point stability / realisability

\section{Introduction}

La modélisation de la corrélation pressiontempérature est une deuxième étape dans la modélisation des corrélations faisant intervenir les fluctuations de pression. Les modèles développés la dernière décennie pour la corrélation pression-déformation n'ont pas connu, à notre connaissance, une telle extension aux phénomènes thermiques. Launder et al. [1], Shih et Lumley [2] et Launder [3] ont développé des modèles de corrélation pression-température en parfaite cohérence avec leurs modèles des corrélations pression-déformation. Les modèles de corrélation pression-déformation plus récents n'ont pas bénéficié, pour la plupart, d'une extension

\footnotetext{
a Auteur correspondant: sgaaliche@yahoo.fr
}

aux phénomènes thermiques. Dans un travail précédent, nous avons proposé une formulation de la corrélation pression-température [4] en cohérence avec le modèle de retour à l'isotropie de la corrélation pression-déformation de Sarkar et Speziale [5].

Dans cet article, nous développons un modèle de retour à l'isotropie de la corrélation pressiontempérature [6,7], en vue de décrire la décroissance d'une turbulence homogène, initialement anisotrope, associée ou non à un gradient moyen de vitesse.

Nous présentons, à la section 2 le modèle de Chung et Kim que nous retenons pour le terme de retour à l'isotropie de la corrélation pression-déformation, ainsi que le modèle que nous proposons pour le terme de retour à l'isotropie de la corrélation pression-température. La section 3 est consacrée à une étude de stabilité des points fixes 
associés au modèle. À la section 4, une étude de réalisabilité du modèle est effectuée en vue de dégager des conditions à imposer aux constantes de ce modèle. À la section 5, nous comparons les résultats déduits du modèle proposé à ceux obtenus à partir du modèle classique. La dernière section comporte une conclusion au travail présenté dans cet article.

\section{Modèle de retour à l'isotropie proposé pour la corrélation pression-température}

Nous nous proposons d'étudier le retour à l'isotropie d'une turbulence homogène, à grands nombres de Reynolds, initialement anisotrope, évoluant sans gradient de vitesse et présentant ou non un gradient moyen de température. Une telle turbulence est décrite, dans le cadre d'une modélisation conventionnelle au second ordre, par les équations exactes de transport des corrélations doubles. Il s'agit des équations d'évolution des tensions de Reynolds $\overline{u_{i} u_{j}}$, des flux thermiques turbulents $\overline{u_{i} \theta}$ et de la variance de la température $\overline{\theta^{2}}$ :

$$
\begin{aligned}
& \frac{\mathrm{d}}{\mathrm{d} t} \overline{u_{i} u_{j}}=\phi_{i j}-\varepsilon_{i j} \\
& \frac{\mathrm{d}}{\mathrm{d} t} \overline{\theta u_{i}}=\phi_{i \theta}+P_{i \theta}-\varepsilon_{i \theta} \\
& \frac{\mathrm{d}}{\mathrm{d} t} \overline{\theta^{2}}=P_{\theta}-2 \varepsilon_{\theta}
\end{aligned}
$$

où $u_{i}$ et $\theta$ sont respectivement les fluctuations de vitesse et de température, $P_{i \theta}$ et $P_{\theta}$ sont les termes de production dus au gradient moyen de température $T_{, k}$ :

$$
\begin{aligned}
& P_{i \theta}=-\overline{u_{i} u_{k}} T_{, k} \\
& P_{\theta}=-2 \overline{\theta u_{k}} T_{, k}
\end{aligned}
$$

$\phi_{i j}$ et $\phi_{i \theta}$ sont les corrélations pression-déformation et pression-température, $\rho$ est la masse volumique :

$$
\begin{aligned}
\phi_{i j} & =-\bar{p}\left(u_{i, j}+u_{j, i}\right) \\
\phi_{i \theta} & =-\frac{\bar{p}}{\rho} \theta_{, i}
\end{aligned}
$$

et enfin $\varepsilon_{i j}, \varepsilon_{i \theta}$ et $\varepsilon_{\theta}$ sont les termes de dissipation dus aux effets moléculaires :

$$
\begin{aligned}
\varepsilon_{i j} & =\nu \overline{u_{i, k} u_{j, k}} \\
\varepsilon_{i \theta} & =(\alpha+\nu) \overline{u_{i, k} \theta_{, k}} \\
\varepsilon_{\theta} & =\alpha \overline{\theta_{, k} \theta_{, k}}
\end{aligned}
$$

$\nu$ est la viscosité cinématique et $\alpha$ la diffusivité thermique du fluide considéré.

À grands nombres de Reynolds et de Péclet, l'isotropie du processus de dissipation conduit simplement à $\varepsilon_{i j}=$ $\frac{2}{3} \varepsilon \delta_{i j}$ et $\varepsilon_{i \theta}=0$.
Le problème central de la modélisation du retour à l'isotropie d'une turbulence homogène consiste en la modélisation des corrélations pression-déformation $\phi_{i j}$ et pression-température $\phi_{i \theta}$. Pour représenter la partie de retour à l'isotropie de la corrélation $\phi_{i j}$, nous retenons le modèle de Chung et Kim [8] :

$$
\begin{aligned}
& \phi_{i j}=\left(2+C_{1}^{\prime}-\frac{1}{7}\left(15 C_{1}^{\prime}-2 C_{2}\right) I_{b}\right. \\
&\left.+\frac{3}{14}\left(3 C_{1}^{\prime}+C_{2}\right)\left(10 I I I_{b}-9 I I_{b}^{2}\right)\right) b_{i j}-\left(C_{2}-\frac{9}{14}\left(3 C_{1}^{\prime}\right.\right. \\
&\left.\left.\quad+C_{2}\right)\left(5 I I_{b}-4 I I I_{b}\right)\right)\left(b_{i k} b_{k j}-\left(\frac{I I_{b}}{3}\right) \delta_{i j}\right)
\end{aligned}
$$

où $C_{1}^{\prime}$ et $C_{2}$ sont deux constantes : $C_{1}^{\prime}=2$ et $C_{2}=$ $19,5\left(1-\frac{5}{R_{\mathrm{et}}}\right)$ [8]. En effet, ce modèle non-linéaire est très intéressant puisqu'il tient compte de l'effet du nombre de Reynolds turbulent $R_{\text {et }}=\frac{4 k^{2}}{9 \nu \varepsilon}$ ( $\varepsilon$ est le taux de dissipation de l'énergie cinétique turbulente $k=\frac{\overline{q^{2}}}{2}$ ). Il satisfait les conditions générales de réalisabilité et traduit de manière satisfaisante l'essentiel des effets non-linéaires mis en évidence expérimentalement et caractérisant le retour à l'isotropie d'une turbulence homogène [8].

Dans ce travail, et dans le but d'assurer la cohérence avec le modèle non-linéaire de Chung et Kim retenu pour traduire l'anisotropie cinématique et en vue d'une meilleure description des effets non-linéaires du retour à l'isotropie, nous proposons un modèle du terme de retour à l'isotropie de la corrélation pression-température suivant un développement à l'ordre cinq du tenseur d'anisotropie $\vec{b}\left(b_{i j}=\frac{\overline{u_{i} u_{j}}}{\overline{q^{2}}}-\frac{\delta_{i j}}{3}\right)$.

À grands nombres de Reynolds et de Péclet, nous retenons $\vec{b}$ et $\overline{u \theta}$ comme arguments tensoriels pour ce modèle qui se présente alors sous la forme générale :

$$
\begin{aligned}
\phi_{i \theta}=\frac{-\varepsilon}{\overline{q^{2}}}\left(\alpha_{1} \overline{\theta u_{i}}+\left(\alpha_{2} b_{i j}+\alpha_{3} b_{i j}^{2}\right.\right. \\
\\
\left.\left.\quad+\alpha_{4} b_{i j}^{3}+\alpha_{5} b_{i j}^{4}+\alpha_{6} b_{i j}^{5}\right) \overline{\theta u_{j}}\right)
\end{aligned}
$$

où $\alpha_{1}, \alpha_{2}, \alpha_{3}, \alpha_{4}, \alpha_{5}$ et $\alpha_{6}$ sont des scalaires dépendant a priori des invariants indépendants qui peuvent être construits à partir de $\vec{b}$ et $\overline{u \theta}$.

Appliquons à cette formulation le théorème de CayleyHamilton.

D'après ce théorème, nous pouvons écrire :

$$
\begin{aligned}
b^{3} & =\frac{1}{2} I I_{b} b+\frac{I I I_{b}}{3} I \\
b^{4} & =\frac{1}{2} I I_{b} b^{2}+\frac{I I I_{b}}{3} b \\
b^{5} & =\frac{1}{2} I I_{b} b^{3}+\frac{I I I_{b}}{3} b^{2}
\end{aligned}
$$


Ces relations permettent d'écrire $\phi_{i \theta}$ sous la forme :

$$
\begin{aligned}
\phi_{i \theta}=-\frac{\varepsilon}{\overline{q^{2}}}\left[\alpha_{1} \overline{u_{i}}+\right. & {\left[\left(\alpha_{2}+\frac{1}{2} I I_{b} \alpha_{4}+\alpha_{5} \frac{I I I_{b}}{3}\right.\right.} \\
\left.+\frac{1}{4} \alpha_{6} I I_{b}^{2}\right) b_{i j} & +\left(\alpha_{3}+\frac{1}{2} \alpha_{5} I I_{b} \alpha_{6} \frac{I I I_{b}}{3}\right) b_{i j}^{2} \\
& \left.\left.+\frac{1}{6} \alpha_{6} I I_{b} I I I_{b}+\alpha_{4} \frac{I I I_{b}}{3}\right] \overline{\theta u_{j}}\right]
\end{aligned}
$$

ou encore :

$$
\phi_{i \theta}=-\frac{\varepsilon}{\overline{q^{2}}}\left(\alpha_{1}^{\prime} \overline{\theta u_{i}}+\left(\alpha_{2}^{\prime} b_{i j}+\alpha_{3}^{\prime} b_{i j}^{2}\right) \overline{\theta u_{j}}\right)
$$

où :

$$
\begin{aligned}
\alpha_{1}^{\prime} & =\alpha_{1}+\alpha_{4} \frac{I I_{b}}{3}+\frac{1}{6} \alpha_{6} I I_{b} I I I_{b} \\
\alpha_{2}^{\prime} & =\alpha_{2}+\frac{1}{2} I I_{b} \alpha_{4}+\alpha_{5} \frac{I I I_{b}}{3}+\frac{1}{4} \alpha_{6} I I_{b}^{2} \\
\alpha_{3}^{\prime} & =\alpha_{3}+\frac{1}{2} \alpha_{5} I I_{b}+\frac{1}{3} \alpha_{6} I I I_{b}
\end{aligned}
$$

À ce stade, il importe de chercher les points fixes du système différentiel des équations d'évolution prenant en compte le modèle proposé (10) et d'étudier la stabilité de tels points fixes.

\section{3 Étude de stabilité des points fixes du système différentiel des équations d'évolution}

Nous nous proposons maintenant d'étudier le retour à l'isotropie d'une turbulence homogène initialement anisotrope évoluant en l'absence de gradients moyens de vitesse et de température et plus précisément, de déterminer les états asymptotiques d'équilibre d'une telle turbulence. L'étude concernant le champ cinématique a été effectuée par Chung et Kim [8]. Il reste à envisager une étude analogue relative au champ thermique. Pour ce faire, nous retenons les invariants scalaires sans dimensions $M_{1}=\frac{\overline{\theta u_{i}}}{\overline{q^{2}}} \overline{\frac{\theta u_{i}}{\theta^{2}}}, \quad M_{2}=\frac{b_{i j} \overline{\theta u_{i}} \overline{\theta u_{j}}}{\overline{q^{2}} \overline{\theta^{2}}}$ et $M_{3}=\frac{b_{i j}^{2} \overline{\theta u_{i}} \overline{\theta u_{j}}}{\overline{q^{2}} \overline{\theta^{2}}}$ pour représenter le champ thermique.

Il s'agit maintenant d'écrire les équations d'évolution de $M_{1}, M_{2}$ et $M_{3}$. En l'absence de gradient moyen de température, et compte tenu du modèle (10) proposé pour $\phi_{i \theta}$, ces équations sont déduites des équations d'évolution de $\overline{\theta u_{i}}, b_{i j}, \overline{\theta^{2}}$ et $\overline{q^{2}}$ qui s'écrivent sous les formes :

$$
\begin{aligned}
\frac{\mathrm{d} \overline{u_{i} \theta}}{\mathrm{d} t}=-\frac{\varepsilon}{\overline{q^{2}}}\left[\alpha_{1} \overline{u_{i}}+\right. & {\left[\left(\alpha_{2}+\frac{1}{2} I I_{b} \alpha_{4}+\alpha_{5} \frac{I I I_{b}}{3}\right.\right.} \\
\left.+\frac{1}{4} \alpha_{6} I I_{b}^{2}\right) b_{i j}+ & \left(\alpha_{3}+\frac{1}{2} \alpha_{5} I I_{b}+\alpha_{6} \frac{I I I_{b}}{3}\right) b_{i j}^{2} \\
& \left.\left.+\frac{1}{6} \alpha_{6} I I_{b} I I I_{b}+\alpha_{4} \frac{I I I_{b}}{3}\right] \overline{\theta u_{j}}\right]
\end{aligned}
$$

$$
\begin{aligned}
\frac{\mathrm{d} b_{i j}}{\mathrm{~d} t}=-\frac{\varepsilon}{\overline{q^{2}}}\left[\left(C_{1}^{\prime}-\frac{1}{7}\left(15 C_{1}^{\prime}-2 C_{2}\right) I I_{b}\right.\right. \\
\left.\quad+\frac{3}{14}\left(3 C_{1}^{\prime}+C_{2}\right)\left(10 I I I_{b}-9 I I_{b}^{2}\right)\right) b_{i j} \\
\left.-\left(C_{2}-\frac{9}{14}\left(3 C_{1}^{\prime}+C_{2}\right)\left(5 I I_{b}-4 I I I_{b}\right)\right)\left(b_{i j}^{2}-\frac{1}{3} I I_{b} \delta_{i j}\right)\right]
\end{aligned}
$$

$$
\begin{aligned}
& \frac{\mathrm{d} \overline{\theta^{2}}}{\mathrm{~d} t}=-2 \varepsilon_{\theta} \\
& \frac{\mathrm{d} \overline{q^{2}}}{\mathrm{~d} t}=-2 \varepsilon
\end{aligned}
$$

Elles conduisent aux équations d'évolution suivantes de $M_{1}, M_{2}$ et $M_{3}$

$$
\begin{aligned}
\frac{\mathrm{d} M_{1}}{\mathrm{~d} \tau}=2[(1+r & \left.-\alpha_{1}-\alpha_{4} \frac{I I I_{b}}{3}-\frac{1}{6} \alpha_{6} I I_{b} I I_{b}\right) M_{1} \\
- & \left(\alpha_{2}+\frac{1}{2} I I_{b} \alpha_{4}+\alpha_{5} \frac{I I_{b}}{3}+\frac{1}{4} \alpha_{6} I I_{b}^{2}\right) M_{2} \\
& \left.-\left(\alpha_{3}+\frac{1}{2} \alpha_{5} I I_{b}+\alpha_{6} \frac{I I I_{b}}{3}\right) M_{3}\right]
\end{aligned}
$$

$$
\begin{aligned}
\frac{\mathrm{d} M_{2}}{\mathrm{~d} \tau}=- & {\left[\left(\frac{1}{3} I I_{b} \alpha_{2}-\frac{3}{14}\left(3 C_{1}^{\prime}+C_{2}\right)\left(5 I I_{b}-4 I I I_{b}\right)\right)\right.} \\
+ & \left.2\left(\alpha_{3}+\frac{1}{2} \alpha_{5} I I_{b}+\alpha_{6} \frac{I I I_{b}}{3}\right) I I_{b}\right] M_{1} \\
& -\left[\left(C_{1}^{\prime}-\frac{1}{7}\left(15 C_{1}^{\prime}-2 C_{2}\right) I I_{b}\right.\right. \\
& \left.+\frac{3}{14}\left(3 C_{1}^{\prime}+C_{2}\right)\left(10 I I I_{b}-9 I I_{b}^{2}\right)\right) \\
-2( & \left.\left.-1-r+\alpha_{1}+\alpha_{4} \frac{I I I_{b}}{3}+\frac{1}{6} \alpha_{6} I I_{b} I I I_{b}\right)\right] M_{2} \\
- & {\left[\left(C_{2}-\frac{9}{14}\left(3 C_{1}^{\prime}+C_{2}\right)\left(5 I I_{b}-4 I I I_{b}\right)\right)\right.} \\
+ & \left.2\left(\alpha_{2}+\frac{1}{2} I I_{b} \alpha_{4}+\alpha_{5} \frac{I I I_{b}}{3}+\frac{1}{4} \alpha_{6} I I_{b}^{2}\right)\right] M_{3}
\end{aligned}
$$

$$
\begin{aligned}
\frac{\mathrm{d} M_{3}}{\mathrm{~d} \tau}=-2\left[\left(C_{2}-\frac{9}{14}\left(3 C_{1}^{\prime}+C_{2}\right)\left(5 I I_{b}-4 I I I_{b}\right)\right.\right. \\
\left.-\alpha_{2}-\frac{1}{2} I I_{b} \alpha_{4}-\alpha_{5} \frac{I I I_{b}}{3}-\frac{1}{4} \alpha_{6} I I_{b}^{2}\right) I I I_{b} \\
\left.\quad+I V_{b}\left(\alpha_{3}+\frac{1}{2} \alpha_{5} I I_{b}+\alpha_{6} \frac{I I I_{b}}{3}\right)\right] M_{1} \\
-\frac{2}{3} I I_{b}\left(\alpha_{2}-\frac{9}{14}\left(3 C_{1}^{\prime}+C_{2}\right)\left(5 I I_{b}-4 I I_{b}\right)\right) M_{2} \\
-2\left[-1-r+\alpha_{1}+\left(\frac{\alpha_{4}}{3}+\frac{\alpha_{6}}{3} I I_{b}\right) I I I_{b}+C_{1}^{\prime}\right. \\
\left.-\frac{1}{7}\left(15 C_{1}^{\prime}-2 C_{2}\right) I I_{b}+\frac{3}{14}\left(3 C_{1}^{\prime}+C_{2}\right)\left(10 I I I_{b}-9 I I_{b}^{2}\right)\right] M_{3}
\end{aligned}
$$


où nous avons utilisé le temps adimensionnel $\tau$ défini par $\mathrm{d} \tau=\frac{\varepsilon}{q^{2}} \mathrm{~d} t$. Ces équations font intervenir $M_{1}, M_{2}, M_{3}$, les invariants $I I_{b}, I I I_{b}$ et le rapport $r$ des temps caractéristiques cinématique et thermique :

$$
r=\frac{\overline{q^{2}} / \varepsilon}{\overline{\theta^{2}} / \varepsilon_{\theta}}
$$

À ce stade, il est nécessaire d'associer à ce système d'équations une équation d'évolution de $r$. Une telle équation peut être obtenue à partir des équations d'évolution (15) et (16) de $\overline{\theta^{2}}$ et $\overline{q^{2}}$ et des équations d'évolution modélisées de $\varepsilon$ et $\varepsilon_{\theta}$. L'équation d'évolution de $\varepsilon$ est écrite sous la forme standard :

$$
\frac{\mathrm{d} \varepsilon}{\mathrm{d} t}=-2 C_{\varepsilon 2} \frac{\varepsilon^{2}}{\overline{q^{2}}} \quad \text { où } \quad C_{\varepsilon 2}=1,9
$$

En ce qui concerne la modélisation de l'équation d'évolution de $\varepsilon_{\theta}$, nous adoptons la forme générale de Newman [9] :

$$
\begin{aligned}
\frac{\mathrm{d} \varepsilon_{\theta}}{\mathrm{d} t}=-C_{D 1} \frac{\varepsilon_{\theta}^{2}}{\overline{\theta^{2}}}-2 C_{D 2} \frac{\varepsilon}{\overline{q^{2}}} \varepsilon_{\theta} \\
\text { où } \quad C_{D 1}=2,02, C_{D 2}=0,89
\end{aligned}
$$

Compte tenu des équations (15), (16), (21) et (22), l'évolution du rapport $r$ en fonction du temps adimensionnel $\tau$ est alors décrite par l'équation :

$$
\frac{1}{r} \frac{\mathrm{d} r}{\mathrm{~d} \tau}=2\left(C_{\varepsilon 2}-C_{D 2}-1\right)+\left(2-C_{D 1}\right) r
$$

Le choix des coefficients est basé sur le bon comportement de $r$, qui doit, en particulier, tendre asymptotiquement $(\tau \rightarrow \infty)$ vers une valeur d'équilibre $r_{\mathrm{e}}(r$ ne doit tendre ni vers zéro ni vers l'infini), soit :

$$
\frac{\mathrm{d}}{\mathrm{d} \tau} r=0 \quad \text { pour } \quad r=r_{\mathrm{e}}
$$

et ceci impose la condition

$$
2\left(C_{\varepsilon 2}-C_{D 2}-1\right)+\left(2-C_{D 1}\right) r_{\mathrm{e}}=0
$$

et permet de réécrire (23) sous la forme :

$$
\frac{1}{r} \frac{\mathrm{d} r_{\mathrm{e}}}{\mathrm{d} \tau}=\left(2-C_{D 1}\right)\left(r-r_{\mathrm{e}}\right)
$$

La recherche des points fixes du système des équations d'évolution de $M_{1}, M_{2}$ et $M_{3}$ est effectuée en remplaçant $r$ par sa valeur asymptotique d'équilibre $r_{\mathrm{e}}$ et en annulant les dérivées $\frac{\mathrm{d}}{\mathrm{d} \tau} M_{1}, \frac{\mathrm{d}}{\mathrm{d} \tau} M_{2}, \frac{\mathrm{d}}{\mathrm{d} \tau} M_{3}$.

Rappelons que Chung et Kim [8] ont mis en évidence, en turbulence cinématique, quatre points fixes. L'unique point fixe stable est caractérisé par $\left(I I_{b}\right)_{\infty}=0,\left(I I I_{b}\right)_{\infty}=$ 0 . Nous remplaçons $\left(I I_{b}\right)_{\infty}$ et $\left(I I I_{b}\right)_{\infty}$ par des valeurs nulles et nous obtenons le système algébrique simplifié suivant :

$(S)$

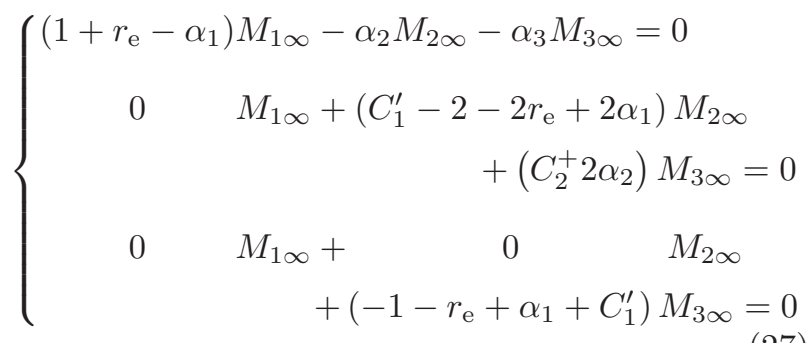

Il s'agit d'un système linéaire homogène en $M_{1 \infty}, M_{2 \infty}$ et $M_{3 \infty}$, à matrice triangulaire supérieure dont la solution unique est :

$$
M_{1 \infty}=0, \quad M_{2 \infty}=0, \quad M_{3 \infty}=0,
$$

à condition que :

$$
\alpha_{1} \neq 1+r_{\mathrm{e}}, \quad 2 \alpha_{1} \neq 2 r_{\mathrm{e}}-C_{1}^{\prime}+2, \quad \alpha_{1} \neq r_{\mathrm{e}}-C_{1}^{\prime}+1 .
$$

L'étude de stabilité [7] des solutions précédentes nous ramène aux conditions finales de stabilité [7] des points fixes :

$$
\begin{aligned}
& \alpha_{1}>r_{\mathrm{e}}+1 \\
& C_{D 1}>2
\end{aligned}
$$

Une première contrainte sur le coefficient $\alpha_{1}$ est obtenue. En vue de dégager des contraintes supplémentaires sur les coefficients du modèle proposé, une étude de réalisabilité est aussi entreprise. Elle fait l'objet de la section 4 .

\section{Application de la condition de réalisabilite au modèle proposé}

L'étude de réalisabilité des modèles au second ordre est un concept fondamental dans la modélisation au second ordre. Elle a été introduite par Shumann [10] et Lumley [11] et étendue par la suite par Sarkar et Speziale [5]. Elle consiste à imposer des contraintes mathématiques pour traduire une réalité physique. Elle a pour objet de préciser les conditions à imposer à un modèle pour que celui-ci ne puisse, en aucune façon, conduire à une prédiction comportant des aberrations physiques (énergie cinétique négative, inégalité de Schwarz non vérifiée pour un coefficient de corrélation).

Pour le champ thermique d'une turbulence homogène, cette condition est imposée à la première composante $D_{11}$ du tenseur symétrique positif $\vec{D}$ de composantes $D_{i j}=$ $\overline{u_{i} \theta} \overline{u_{j} \theta}-\overline{\theta^{2}} \overline{u_{i} u_{j}}$ introduit par Lumley [11]. D'une manière générale, une condition suffisante de réalisabilité sur une quantité positive $F$ est écrite sous la forme :

$$
\frac{\mathrm{d} F}{\mathrm{~d} t} \geqslant 0 \text { quand } F \rightarrow 0 .
$$

Pour satisfaire une telle condition, nous écrivons l'équation d'évolution de $D_{11}$ en introduisant le modèle 
de Chung et Kim (7) pour $\phi_{i j}$ et le modèle proposé (10) pour $\phi_{i \theta}$ :

$$
\begin{gathered}
\frac{\mathrm{d} D_{11}}{\mathrm{~d} t}=-2 \varepsilon_{\theta} \overline{u_{1}^{2}}-\varepsilon \overline{\theta^{2}}\left[\left(C_{1}^{\prime}-\frac{1}{7}\left(15 C_{1}^{\prime}-2 C_{2}\right) I I_{b}\right.\right. \\
\left.+\frac{3}{14}\left(3 C_{1}^{\prime}+C_{2}\right)\left(10 I I I_{b}-9 I I_{b}^{2}\right)\right) b_{11} \\
\left.-\left(C_{2}-\frac{9}{14}\left(3 C_{1}^{\prime}+C_{2}\right)\left(5 I I_{b}-4 I I I_{b}\right)\right)\left(b_{11}^{2}-\frac{1}{3} I I_{b}\right)\right]+2 \overline{\theta u_{1}} \frac{\varepsilon}{\overline{q^{2}}} \\
\times\left[\left(\alpha_{1}+\alpha_{4} \frac{I I I_{b}}{3}+\frac{1}{6} \alpha_{6} I I_{b} I I I_{b}\right) \overline{\theta u_{1}}\right. \\
+\left(\alpha_{2}+\frac{1}{2} I I_{b} \alpha_{4}+\alpha_{5} \frac{I I I_{b}}{3}+\frac{1}{4} \alpha_{6} I I_{b}^{2}\right) b_{11} \overline{\theta u_{1}} \\
\left.+\left(\alpha_{3}+\frac{1}{2} \alpha_{5} I I_{b}+\frac{1}{3} \alpha_{6} I I I_{b}\right) b_{11}^{2} \overline{\theta u_{1}}\right]
\end{gathered}
$$

qui s'écrit de manière synthétique sous la forme suivante :

$$
\frac{\mathrm{d} D_{11}}{\mathrm{~d} t}=A M+B
$$

où $A$ et $B$ sont des fonctions compliquées des invariants $I I_{b}, I I I_{b}, \overline{I_{D}}, \overline{I I_{D}}$ et des constantes numériques $C^{\prime}{ }_{1}, C_{2}$ et $\alpha_{1}, \alpha_{2}, \alpha_{3}, \alpha_{4}, \alpha_{5}, \alpha_{6}$.

Et si nous imposons la condition $\frac{\mathrm{d}}{\mathrm{d} t} D_{11} \geqslant 0$, quand $D_{11}=0$, nous obtenons

$$
A M+B \geqslant 0
$$

où les coefficients $A$ et $B$ sont de la forme :

$$
\begin{aligned}
& A= {\left[-2 r_{\mathrm{e}}+2\left(\alpha_{1}+\alpha_{4} \frac{I I I_{b}}{3}+\frac{1}{6} \alpha_{6} I I_{b} I I I_{b}\right)\right.} \\
&-\left(C_{1}^{\prime}-\frac{1}{7}\left(15 C_{1}^{\prime}-2 C_{2}\right) I I_{b}+\frac{3}{14}\left(3 C_{1}^{\prime}+C_{2}\right)\left(10 I I I_{b}-9 I I_{b}^{2}\right)\right) \\
&+\left(\frac{1}{3}-\overline{I_{D}}\right)\left(C_{2}-\frac{9}{14}\left(3 C_{1}^{\prime}+C_{2}\right)\left(5 I I_{b}-4 I I I_{b}\right)\right) \\
&+2\left(\frac{2}{3}-\overline{I_{D}}\right)\left(\alpha_{2}+\frac{1}{2} I I_{b} \alpha_{4}+\alpha_{5} \frac{I I I_{b}}{3}+\frac{1}{4} \alpha_{6} I I_{b}^{2}\right) \\
& \\
&\left.+2\left(\frac{1}{9}-I I_{b}-\frac{\overline{I_{D}}}{3}+\overline{I I}_{D}\right)\left(\alpha_{3}+\frac{1}{2} \alpha_{5} I I_{b}+\alpha_{6} \frac{I I I_{b}}{3}\right)\right]
\end{aligned}
$$

$$
\begin{aligned}
B=\varepsilon \overline{\theta^{2}}\left[\frac { 1 } { 3 } \left(C_{1}^{\prime}-\frac{1}{7}\left(15 C_{1}^{\prime}-2 C_{2}\right) I I_{b}\right.\right. \\
\left.\quad+\frac{3}{14}\left(3 C_{1}^{\prime}+C_{2}\right)\left(10 I I I_{b}-9 I I_{b}^{2}\right)\right) \\
\left.-\frac{2}{9}\left(C_{2}-\frac{9}{14}\left(3 C_{1}^{\prime}+C_{2}\right)\left(5 I I_{b}-4 I I I_{b}\right)\right)\right]
\end{aligned}
$$

Une analyse de la quantité $B$, à travers le diagramme de réalisabilité de Lumley [11] (voir annexe), a montré que son signe dépend du coefficient $C_{2}$ du terme de retour à l'isotropie de la corrélation pression-déformation et elle est positive pour la valeur optimale de $C_{2}$. Une condition suffisante de la réalisabilité est alors obtenue lorsqu'on a :

$$
B>0 \quad \text { et } A=0
$$

En considérant la base formée des invariants indépendants $\overline{I_{D}}$ et $\overline{I I_{D}}, A$ peut se mettre sous la forme $A=A_{0}+$ $A_{1} \overline{I_{D}}+A_{2} \overline{I I_{D}}$, ce qui nous ramène à la condition $A_{0}=$ $A_{1}=A_{2}=0$.

Nous obtenons dans ces conditions le système algébrique suivant :

$$
\alpha_{3}+\frac{1}{2} \alpha_{5} I I_{b}+\alpha_{6} \frac{I I I_{b}}{3}=0
$$

$$
\begin{aligned}
\left(C_{2}-\frac{9}{14}\left(3 C_{1}^{\prime}+C_{2}\right)\left(5 I I_{b}-4 I I I_{b}\right)\right) \\
-2\left(\alpha_{2}+\frac{1}{2} I I_{b} \alpha_{4}+\alpha_{5} \frac{I I I_{b}}{3}+\frac{1}{4} \alpha_{6} I I_{b}^{2}\right) \\
-\left(\alpha_{3}+\frac{1}{2} \alpha_{5} I I_{b}+\alpha_{6} \frac{I I I_{b}}{3}\right)=0
\end{aligned}
$$

$$
\begin{aligned}
-2 r_{\mathrm{e}}+2\left(\alpha_{1}+\alpha_{4} \frac{I I I_{b}}{3}+\frac{1}{6} \alpha_{6} I I_{b} I I I_{b}\right) \\
-\left(C_{1}^{\prime}-\frac{1}{7}\left(15 C_{1}^{\prime}-2 C_{2}\right) I I_{b}\right. \\
\left.+\frac{3}{14}\left(3 C_{1}^{\prime}+C_{2}\right)\left(10 I I I_{b}-9 I I_{b}^{2}\right)\right) \\
+\frac{1}{3}\left(C_{2}-\frac{9}{14}\left(3 C_{1}^{\prime}+C_{2}\right)\left(5 I I_{b}-4 I I I_{b}\right)\right) \\
+\frac{4}{3}\left(\alpha_{2}+\frac{1}{2} I I_{b} \alpha_{4}+\alpha_{5} \frac{I I I_{b}}{3}+\frac{1}{4} \alpha_{6} I I_{b}^{2}\right) \\
+2\left(\frac{1}{9}-I I_{b}\right)\left(\alpha_{3}+\frac{1}{2} \alpha_{5} I I_{b}+\alpha_{6} \frac{I I I_{b}}{3}\right)=0
\end{aligned}
$$

qui se ramène à la forme suivante si (40) est introduite dans (41) et (41) dans (42)

$$
\begin{aligned}
\alpha_{3}+\frac{1}{2} \alpha_{5} I I_{b} & +\alpha_{6} \frac{I I I_{b}}{3}=0 \\
\alpha_{2}+\frac{1}{2} I I_{b} \alpha_{4} & +\alpha_{5} \frac{I I I_{b}}{3}+\frac{1}{4} \alpha_{6} I I_{b}^{2}= \\
& -\frac{1}{2}\left(C_{2}-\frac{9}{14}\left(3 C_{1}^{\prime}+C_{2}\right)\left(5 I I_{b}-4 I I I_{b}\right)\right) \\
\alpha_{1}+\alpha_{4} \frac{I I I_{b}}{3}+ & \alpha_{6} \frac{1}{6} I I_{b} I I I_{b}= \\
r_{\mathrm{e}} & +\frac{1}{2}\left(C_{1}^{\prime}-\frac{1}{7}\left(15 C_{1}^{\prime}-2 C_{2}\right) I I_{b}\right. \\
& \left.+\frac{3}{14}\left(3 C_{1}^{\prime}+C_{2}\right)\left(10 I I I_{b}-9 I I_{b}^{2}\right)\right)
\end{aligned}
$$

Nous devons signaler ici que les membres gauches de ces trois égalités représentent les coefficients $\alpha_{3}^{\prime}, \alpha_{2}^{\prime}$ et $\alpha_{1}^{\prime}$ 
du troisième, deuxième et premier terme de la forme (11) proposée pour la corrélation pression-température et nous obtenons alors :

$$
\begin{aligned}
& \alpha_{3}^{\prime}= \\
& \alpha_{2}^{\prime}=-\frac{1}{2}\left(C_{2}-\frac{9}{14}\left(3 C_{1}^{\prime}+C_{2}\right)\left(5 I I_{b}-4 I I I_{b}\right)\right) \\
& \alpha_{1}^{\prime}=r_{\mathrm{e}}+\frac{1}{2}\left(C_{1}^{\prime}-\frac{1}{7}\left(15 C_{1}^{\prime}-2 C_{2}\right) I_{b}\right. \\
&\left.\quad+\frac{3}{14}\left(3 C_{1}^{\prime}+C_{2}\right)\left(10 I I I_{b}-9 I I_{b}^{2}\right)\right)
\end{aligned}
$$

Et la nouvelle expression du modèle proposé pour $\phi_{i \theta}$ se présentera sous la forme :

$$
\begin{gathered}
\phi_{i \theta}=-\frac{\varepsilon}{\overline{q^{2}}}\left[r_{\mathrm{e}}+\frac{1}{4}\left(C_{1}^{\prime}-\frac{1}{7}\left(15 C_{1}^{\prime}-2 C_{2}\right) I I_{b}\right.\right. \\
\left.+\frac{3}{14}\left(3 C_{1}^{\prime}+C_{2}\right)\left(10 I I I_{b}-9 I I_{b}^{2}\right)\right) \overline{\theta u_{i}} \\
\left.-\frac{1}{2}\left(C_{2}-\frac{9}{14}\left(3 C_{1}^{\prime}+C_{2}\right)\left(5 I I_{b}-4 I I I_{b}\right)\right) b_{i j} \overline{\theta u_{j}}\right]
\end{gathered}
$$

où $C_{1}^{\prime}=2$ et $C_{2}=19,5\left(1-\frac{5}{R_{\mathrm{et}}}\right)$ [8].

Le résultat obtenu est à notre sens intéressant. Il permet d'exprimer les coefficients du terme de retour à l'isotropie de la corrélation pression-température en fonction des coefficients du terme de retour à l'isotropie de la corrélation pression-déformation de Chung et Kim [8], sans avoir recours à l'optimisation numérique des coefficients de ce modèle. Nous comparons à la section suivante les résultats prédits par le modèle de Chung et Kim étendu aux phénomènes thermiques, à ceux des simulations numériques directes de Iida et Kasagi [12] d'une part, et à ceux relatifs au modèle classique de Rotta [13] d'autre part.

\section{Présentation des résultats}

Nous commençons par présenter les résultats relatifs au champ cinématique. Nous nous proposons de comparer les évolutions des grandeurs turbulentes (composantes $\overline{u_{2}^{2}}$ et $\overline{u_{3}^{2}} \mathrm{du}$ tenseur de Reynolds et énergie cinétique turbulente $k$ ) prédites par le modèle de Chung et Kim aux résultats de simulation numérique directe (SND) de Iida et Kasagi [12]. Sont présentées aussi les évolutions prédites par le modèle classique de Rotta [13]. La simulation numérique de l'écoulement étudié [12] est effectuée en utilisant la méthode de Runge-Kutta du quatrième ordre partant de conditions initiales isotropes.

Sur les figures $1 \mathrm{a}$ et $1 \mathrm{~b}$, nous représentons respectivement les évolutions temporelles des composantes $\overline{u_{2}^{2}}$ et $\overline{u_{3}^{2}}$ du tenseur de Reynolds. Nous pouvons remarquer que, contrairement aux résultats de simulation utilisant le modèle de Chung et Kim pour $\phi_{i j}$ montrant une bonne concordance avec les résultats de SND de Iida et Kasagi,

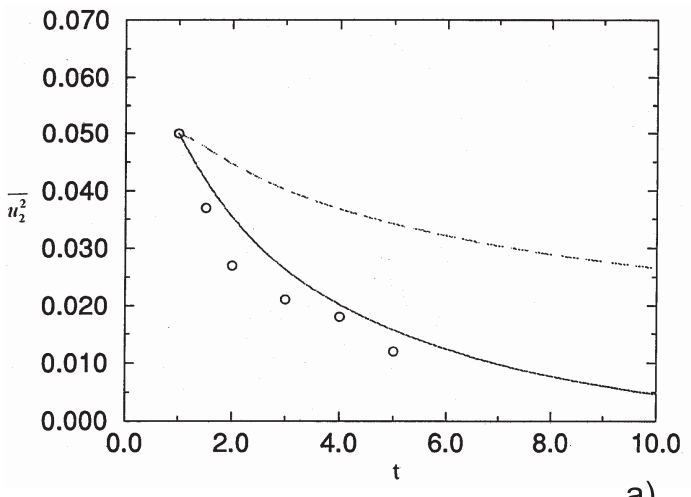

a)

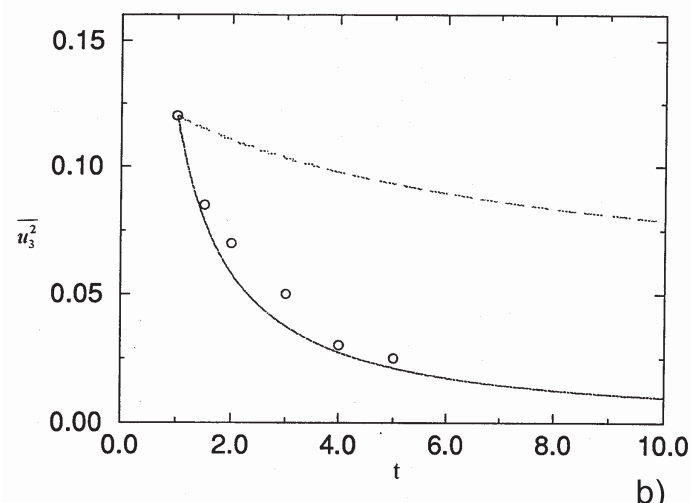

Fig. 1. Évolution de la composante du tenseur de Reynolds $\overline{u_{2}^{2}}$ a). Évolution de la composante du tenseur de Reynolds $\overline{u_{3}^{2}}$ b). o Simulation numérique directe de Iida et Kasagi; - modèle de Chung et Kim ; -- modèle de Rotta.

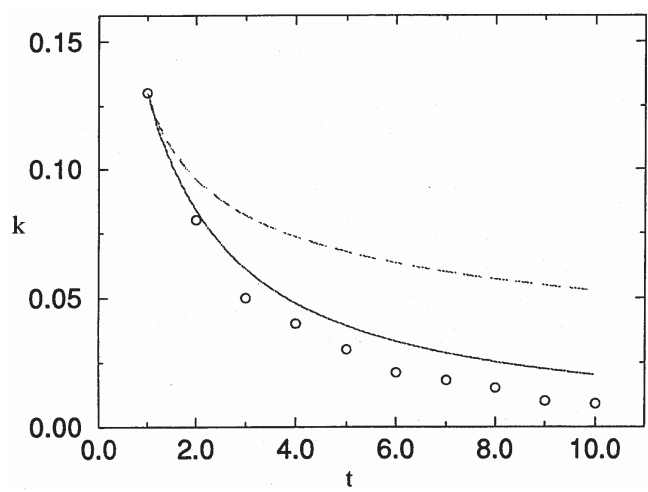

Fig. 2. Évolution de l'énergie cinétique turbulente $k$. o Simulation numérique directe de Iida et Kasagi ; — modèle de Chung et Kim ; -- modèle de Rotta.

les résultats de simulation utilisant le modèle classique de Rotta présentent des écarts nets par rapport aux résultats de Iida et Kasagi. La figure 2 présente l'évolution de l'énergie cinétique turbulente $k$ en fonction du temps. Nous observons que le modèle de Chung et Kim montre là aussi un meilleur accord avec les résultats de simulation numérique directe [12].

Nous allons présenter maintenant les résultats de simulation numérique utilisant la forme finale (44) du modèle de retour à l'isotropie de la corrélation 


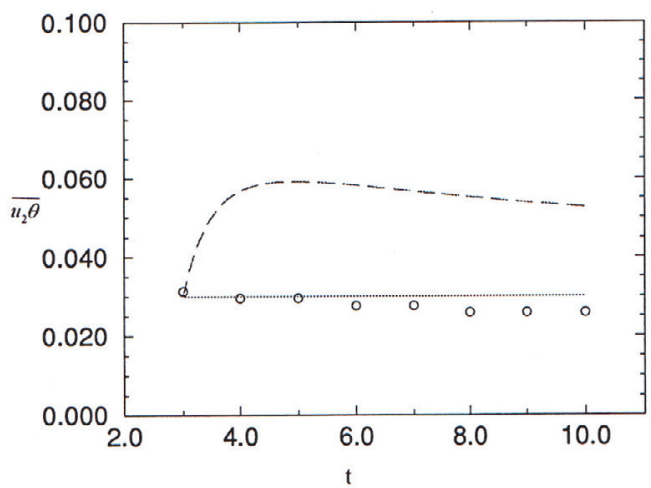

a)

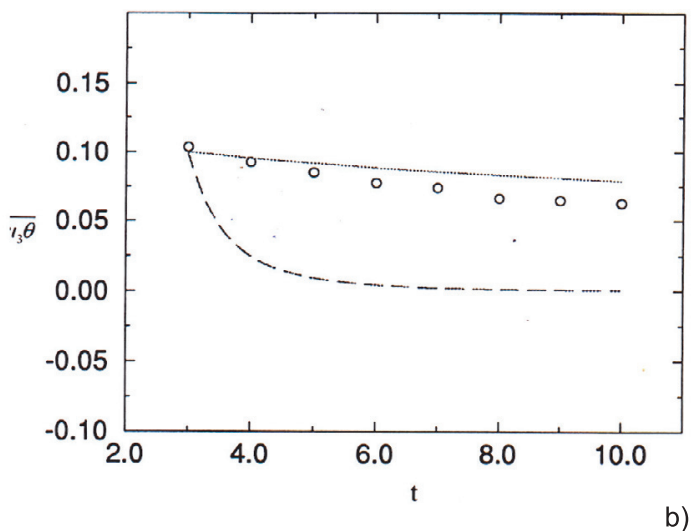

Fig. 3. Évolution de la composante $\overline{u_{2} \theta}$ du flux thermique turbulent a). Évolution de la composante $\overline{u_{3} \theta}$ du flux thermique turbulent b). o simulation numérique directe de Iida et Kasagi : ... modèle proposé : -- modèle de Rotta.

pression-température que nous avons proposé et les valeurs optimales des coefficients $C_{1}^{\prime}$ et $C_{2}$ [8]. Les figures 3a et $3 \mathrm{~b}$ montrent l'évolution des flux thermiques turbulents $\overline{u_{2} \theta}$ et $\overline{u_{3} \theta}$ en fonction du temps $t$. Les résultats de simulation utilisant le modèle proposé sont en très bon accord avec les résultats de simulation numérique directe [12]; des écarts nets sont par contre observés sur ces figures entre les résultats de simulation numérique utilisant le modèle de Rotta [13] et les résultats de simulation numérique directe [12]. Le modèle proposé montre alors une nette amélioration par rapport aux résultats du modèle classique de Rotta.

\section{Conclusion}

Nous avons proposé dans cette étude un modèle non-linéaire de retour à l'isotropie de la corrélation pression-température en parfaite cohérence avec le modèle non-linéaire de Chung et Kim de la corrélation pressiondéformation. À travers une représentation tensorielle isotrope de cette corrélation en fonction du tenseur d'anisotropie $\vec{b}$ et du vecteur flux thermique turbulent $\bar{u} \theta$, nous avons pris l'option d'inclure dans ce modèle des termes d'ordre inférieur ou égal à cinq par rapport au tenseur $\vec{b}$, en vue de permettre la possibilité de prise en compte directe des effets non-linéaires de la turbulence.
Par la suite, l'essentiel de cette contribution a consisté à dégager les contraintes qu'il y a lieu d'imposer au modèle.

Nous avons commencé à la section 3 par examiner la stabilité des points fixes d'une turbulence initialement anisotrope évoluant en l'absence de gradients moyens. Nous avons montré l'existence d'un seul point fixe qui correspond à des valeurs nulles de trois scalaires indépendants $M_{1}, M_{2}$ et $M_{3}$ construits à partir du tenseur d'anisotropie $\vec{b}$ et du vecteur flux thermique turbulent $\bar{u} \theta$. La condition de stabilité de cette solution est assurée par une condition simple sur un coefficient du modèle proposé (44).

En ce qui concerne la réalisabilité des flux thermiques turbulents qui a fait l'objet de la section 4, nous avons suivi la démarche de Lumley [11] en introduisant le concept général de réalisabilité par l'intermédiaire d'un tenseur positif $\vec{D}$ construit à partir du tenseur de Reynolds, du vecteur flux thermique turbulent et de la variance de la température. Nous avons pu déterminer une condition suffisante de réalisabilité du modèle proposé en exprimant ses coefficients en fonction des deux coefficients du modèle de retour à l'isotropie de la corrélation pression-déformation et sans avoir recours à une nouvelle optimisation des coefficients.

La section 5 a été consacrée à la présentation des résultats déduits de ce modèle comparés à ceux des simulations numériques directes de Iida et Kasagi d'une part, et à ceux obtenus par le modèle classique d'autre part.

Le modèle proposé a conduit à des résultats qui, comparés aux données de simulations numériques directes de Iida et Kasagi [12], montrent une nette amélioration de la prédiction des flux thermiques turbulents par rapport au modèle classique de Rotta [13].

\section{Annexe}

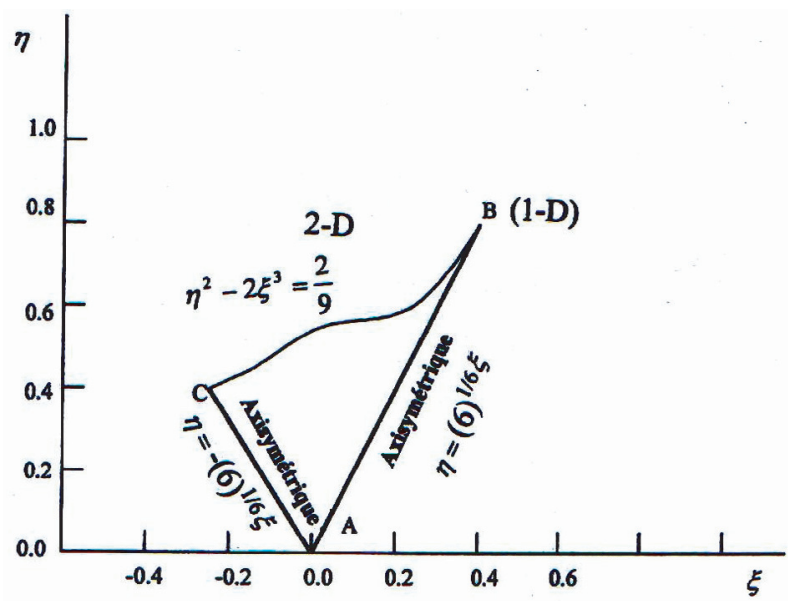

Fig. A.1. Diagramme de réalisabilité de Lumley $\left(\eta=I I_{b}^{1 / 2}\right.$, $\left.\xi=I I I_{b}^{1 / 3}\right)$. 
Les épreuves n'ont pas été relues par les auteurs.

\section{Références}

[1] B.E. Launder, G. Reece, W. Rodi, Progress in the development of a Reynolds stress closure, J. Fluid Mech. 68 (1975) 537

[2] T.H. Shih, J.L. Lumley, Modeling of pressure correlation terms in Reynolds stress and scalars flux equation, Report No FDA-85-3, Cornell University, 1985

[3] B.E. Launder, Topics in Applied Physics, P. Bradshaw (éd.), 12, 1976

[4] M. Bouzaiane, T. Lili, Réalisabilité d'un schéma nonlinéaire de retour à l'isotropie de la corrélation pressiongradient d'un scalaire, Société Tunisienne de Physique, Hammamet, mars 1995, pp. 267-272

[5] S. Sarkar, C.G. Speziale, A simple non-linear model for the return to isotropy in turbulence, Phys. Fluids A 2 (1990) 84

[6] M. Bouzaiane, S. Gaaliche, T. Lili, Réalisabilité d'un modèle non-linéaire de la corrélation pression-température, $16^{\text {e }}$ Congrès Français de Mécanique, Nice, 1-5 septembre 2003

[7] S. Gaaliche, M. Bouzaiane, T. Lili, Extension du modèle de Chung et Kim, à la corrélation pression-température : Étude de réalisabilité, $4^{\text {es }}$ Journées Tunisiennes sur les Écoulements et les Transferts, Hammamet, 2123 décembre 2002

[8] M.K. Chung, S.K. Kim, A non linear return-to-isotropy model with Reynolds number and anisotropy dependency, Phys. Fluids 7 June 1995

[9] G.R. Newman, B.E. Launder, J.L. Lumley, Modeling the behaviour of homogeneous scalar turbulence, J. Fluid Mech. 111 (1981) 217

[10] U. Shumann, Realisability of Reynolds-stress turbulence models, Phys. Fluids 20 (1977) 721-728

[11] J.L. Lumley, Computational modelling of turbulent flows, Adv. Appl. Mech. 18 (1978) 123

[12] O. Iida, N. Kasagi, 9th Symposium Turbulent Shear Flows, Kyoto, Japan, August 16-18, 1993

[13] J.C. Rotta, Statisahe theorie nichthomogener turbulenze, I. Mitteilung, Z. Phys. 129 (1951) 547 